\title{
DE LA INVOCACIÓN AL PARALELISMO: EL AUTORRETRATO INICIAL DE LEÓN FELIPE EN ROCINANTE
}

\author{
Jorge Chen Sham
}

\begin{abstract}
RESUMEN
En este artículo, el autor analiza la revalorización del poeta realizada en el poemario Rocinante, de León Felipe. Lo inédito del análisis ofrecido en Rocinante estriba en la total integración entre la poesía y la vida, a partir de una indagación que cobra nuevo sentido.

A.STKACT

The author analizes the poet's revaluation in León Felipe's poem collection Rocinante. The novelty in the analysis presented in Rocinante lies in the total integration between poetry and life that stems from a type of questioning that acquires a new meaning.
\end{abstract}

En el poemario Rocinante, empezado en 1965 pero publicado póstumamente (México D.F.: Finisterre, 1968), León Felipe responde, en forma recapituladora y en la madurez de su existencia, al recorrido iniciático que enunciaba en el subtítulo de Ganarás la luz (primera edición de 1943): "Biografía, Poesía, Destino". Se trata, en palabras de Tomás Rivera, de un proceso iluminativo en el que "la poesía es principalmente el anhelo por identificarse y resolver o preguntar sobre los misterios circundantes" (1973: 205). Sin embargo, lo inédito del análisis ofrecido en Rocinante estriba en la total integración entre la poesía y la vida, a partir de una indagación que cobra ahora nuevo sentido. La condición de poeta se revalora en un diálogo paralelo e intercruzado con un personaje de papel que, a los ojos de León Felipe, se humaniza y adquiere existencia independiente. En ese espacio de la escritura poética, la literatura emerge como símbolo que puede transformar la vida, ya que la aparición de Rocinante sirve de engarce a un universo que irradia su luz y esclarece las cosas gracias a su presencia espiritual, como vio Unamuno por cierto en don Quijote: "su poderosa vitalidad y lo realísimo de su realidad" (Unamuno 1966: 1231) esclarecen la biografía y el destino del yo poeta. Y en ese diálogo con Rocinante, el destino del hablante lírico alcanza, gracias a esa aspiración a la completud, una dimensión ardiente y creadora que devela el sentido del universo (Ortega y Gasset 1990: 75).

Desde este punto de vista, es interesante que León Felipe no convoque, en primer lugar, las figuras ni de don Quijote ni Sancho Panza para establecer el parangón; todo lo contrario, escoge a su interlocutor en la figura un poco marginal y de segundo plano de Rocinante, si tomamos en cuenta que la comparación no se hace con alguno de los personajes principales del 
Quijote. Claro está, la selección no es gratuita como veremos más adelante y cobra su significación en el centro medular del poemario intitulado "Biografías Paralelas", cuyas resonancias con las Vidas paralelas del escritor helenístico Plutarco no se hacen esperar. En un primer momento, el poemario podría comprenderse como la puesta en paralelo de dos vidas, la de Rocinante y la de León Felipe; pero esta experiencia que descentra al poeta poniendo su mirada sobre su existencia se realiza bajo el foco discriminante de un destino poético (Chen 1998: 24), que el sujeto ha sabido forjarse. Interpretado de este modo su existencia, aparece la autobiografía para modelar la visión que tiene el poeta de su camino de aprendizaje ${ }^{1}$, con lo cual expone "la génesis de la situación actual [y] señala [...] una vía -a veces sinuosa- que conduce al estado actual del conocimiento recapitulador" (Starobinski 1974: 72, la cursiva es del autor).

A la luz de lo anterior, Rocinante se transforma en una autointerpretación del proceso autobiográfico y nos revela a un sujeto que se sitúa en esa apremiante necesidad de comprenderse a sí mismo en los otros. Tejiendo así un valor diferencial, León Felipe juzga su biografía a partir de la sabiduría que proporciona la madurez de su largo camino por la vida y, sobre todo, a partir de su situación presente de poeta consagrado: ha obtenido la fama y la notoriedad en la vejez, tal y como ha sucedido también con Rocinante, un jamelgo desdentado que ha saltado a la gloria literaria como él. La comparación se vuelve aquí certitud del yo (Beaujour 1980: 451) y el poemario se abre con un "Saludo", un poema-invocación con todas las características de las clásicas epístolas dedicatorias en las que se rendía un homenaje al protector, a quien se dedicaba la obra buscando en él un garante de la misma (Genette 1987: 114-5). En este caso, León Felipe se dirige a Rocinante y le otorga gran relevancia como personaje que merece todo su respeto y admiración:

\footnotetext{
¡iYo te saludo, Rocinante!!

¡Viejo caballo sin estirpe!...

¡no tienes pedigree!
}

Concretando el objeto de su discurso, el hablante lírico hace presente a Rocinante en un intradiálogo que lo hace accesible a la comunicación. El recurso de las constantes interjecciones, en estos versos iniciales, no solo nos introduce en la interioridad anímica de un sujeto que habla con profunda solemnidad, sino que también explora esa necesidad de establecer el contacto. En el encuentro que crea el circuito de comunicación, "[1]a manifestación lírica se realiza en la excitación de este influjo recíproco" (Kayser 1981: 446), tensando las relaciones entre el "yo" y el "tú" bajo una emoción profunda que embarga al hablante lírico. Sin embargo, pareciera que "Saludo" no se ajusta al elogio particularizante de las dedicatorias, pues en lugar de ponderar las cualidades del homenajeado, el poema empieza por su rebajamiento. El yo reconoce en Rocinante la carencia de una genealogía o raíz familiar alguna que le nombre (v.2), reforzado por el sustantivo "pedigree", documento que confirma el linaje de un animal y la herencia de sus antepasados. Esta aparente contradicción desencadena el acto de anagnórisis por parte del hablante lírico (v.4), con una afirmación que traduce la sorpresa ante el atípico saludo inicial, en donde la objetividad del enunciado contrasta con su negación subjetiva:

¡Oh Dios mío, Rocinante no tiene pedigree!

Historiadores, eruditos, detectives, (v.5)

traperos,

buscadores de pañales y baberos blasonados...

¡Rocinante no tiene pedigree! 
Hay que recordar que en el mismo Quijote, aparece esta contradicción de planos. En el texto cervantino, la realidad se superpone a la intencionalidad de don Quijote estableciéndose un desfase entre la condición de su caballo, un rocín sin dientes, y el nombre pomposo y lumínico que escogió ${ }^{2}$. Pero también este efecto contrastivo es esencial aquí, porque León Felipe se refiere al animal más famoso de la literatura en lengua española. La situación comunicativa de "Saludo" presupone entonces ese destino caballeresco que don Qujote ha creado para un caballo viejo, del anonimato de su existencia apacible hacia la fama que catapulta los ideales de su amo y, de ahí, hacia el reconocimiento ulterior. Por ello, Rocinante entra en la gloria literaria gracias a este deseo de don Quijote de hacerse caballero (Núñez 1965: 30-4), lo cual se opone al desconocimiento de su linaje. Por eso invita a todos los estudiosos, encargados de llenar este vacío, a un reto intelectual, que busquen y desempolven, en los vestigios de infancia, las pruebas de legitimidad social (v.7) y los signos de su ascendencia nobiliaria ("baberos blasonados"). La respuesta a la solicitud cae por su peso y la afirmación del verso 8 se transforma en una irremediable constatación que nos devuelve al inicio del poema. Sin embargo, en "Saludo" se produce una inversión a esta situación carencial imponiéndose el recorrido propio de todo elogio particularizante. La acción reivindicativa del acto de homenaje surge para acabar, de una vez por todas, con este origen oscuro y con la incertidumbre del pasado ante la luminosidad de su situación presente:

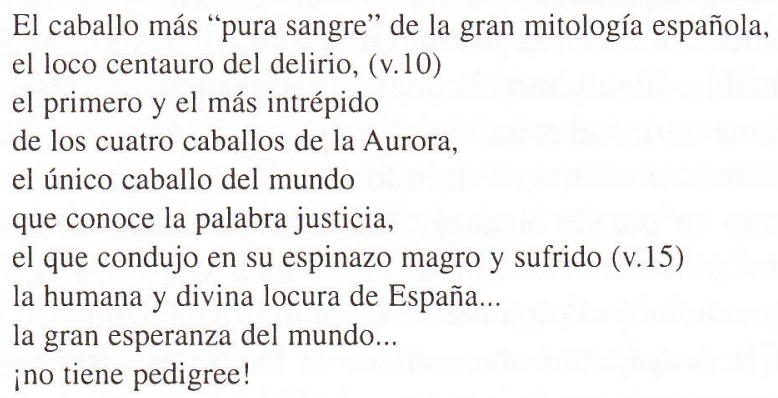

Construida bajo la forma de una aposición en cadena, el resto del poema constituye la respuesta que termina con la falta de un origen noble, gracias a la afirmación que se produce en el v.9. De esta manera, el elogio particularizante justifica las razones por las cuales se le otorga a Rocinante el calificativo de "pura sangre" (v.9), cuando éste únicamente se utilizaría para caballos que posean "pedigree", es decir, ascendencia y linaje sin mancha. La carencia produce el efecto contrario a la exclusión social de Rocinante, pues sirve para legitimar su posición actual. Para ello, se produce en "Saludo" una elipsis de tipo ideológico que destruye la aceptación de un sistema basado en el origen estamental, con el fin de imponer uno nuevo. Tal desplazamiento se obseva en la contraposición que realiza el hablante lírico entre el origen oscuro de Rocinante y su situación presente. Lo anterior presupone también que la motivación del hablante lírico va más allá de la simple exaltación de un personaje, pues lo presenta como modelo de vida, con lo cual la identificación con Rocinante es total y plena y lo eleva a una categoría paradigmática; principio de estructuración que Plutarco sigue en sus Vidas paralelas:

[...] para él la historia constituye sólo la perspectiva, el escenario en el cual los hombres son vistos y a la vez la justificación de su dignidad, de su asunción a una categoría humana superior y 
casi ideal. Para entrar en ella no basta haber sido creadores de historia [...] sino que el personaje histórico, una vez entrado en la historia, puede decirse que se purifica del aspecto contingente de valor paradigmático absoluto [...] (Cantarella 1972: 223-4)

Esta transformación se produce en el Rocinante felipiano y marca su ascenso a una posición más elevada dentro de la "mitología española" (v.9). Jean Chevalier y Alain Gheerbrant señalan que, en el ámbito de los símbolos, el caballo pertenece también al mundo de lo solar ${ }^{3}$. Cuando este caballo se inserta dentro de una epifanía celeste, como sucede en el poema de León Felipe, se intensifica el sentido de la conquista espiritual que alcanza todo iniciado (1993: 216); por eso el hablante lírico ubica a Rocinante en los cielos. Con ello, el poema introduce el motivo de la ascensión y presenta al caballo de don Quijote tirando de la cuádriga de Apolo, que irradia luz y genera una fuerza renovadora para el mundo:

El caballo de majestad. Solar, enganchado al carro del astro, el caballo blanco llega a ser la imagen de la belleza alcanzada por el reinado del espíritu (Chevalier 1993: 216, la cursiva es del autor).

Emplazado en lo alto, Rocinante culmina, entonces, el movimiento que lo catapulta hacia la fama literaria y que lo desplaza de la tierra de los mortales hacia la morada de los dioses. En primer lugar, el hablante lírico potencializa la conquista del espíritu y de valores del idealismo al evocar el texto cervantino; insiste en la fusión entre animal y amo, mediante la figura hipomorfa del "centauro", mitad hombre y mitad caballo. Parafraseando a Salvador de Madariaga cuando postula la complementariedad psicológica entre amo y escudero, la evolución de jinete y caballo aparecen tan íntimamente entrelazados (1972: 159) que son también una unidad; por eso actúan al unísono en pos de alcanzar "las altas verdades de la de Don Quijote, basadas en vida fundamental y honda" (Unamuno 1990: 283; Cap. XXXI, Primera Parte) y el hablante lírico los ve como unidad y complemento en la figura del centauro. Porque don Quijote existe cuando obra, en la concepción unamuniana la locura es vista como salvación que redime al caballero andante y se le otorga el motor de la acción quijotesca:

Llenósele la fantasía de hermosos desatinos, y creyó ser verdad lo que es sólo hermosura. Y lo
creyó con fe tan viva, con fe tan engendradora de obras, que acordó poner en hecho lo que su
desatino le mostraba, y en puro creerlo hízolo verdad (Unamuno 1990: 163; Cap. I, Primera Parte).

Esta "locura" de la que nos habla Unamuno es la que León Felipe avisora en su poema, a la hora de ensalzar ese ideal que se concreta como "la gran esperanza del mundo" (v.17) en tanto el quijotismo sigue inspirando y fortaleciendo los espíritus.

A la luz de una voluntad que se impone como finalidad de vida, emerge Rocinante en posesión de esta verdad quijotesca, como el caballo más privilegiado del orbe. Y si Rocinante puede elevarse al cielo y conducir como caballo resplandecido en blancura el carro solar (vv. $11-2)^{4}$, también hará lo mismo con don Quijote. Por eso, existe en el poema una lógica que desplaza e imbrica las cualidades del amo a su animal, de manera que Rocinante aparece, para el hablante lírico, como la personificación de la "justicia" (v.14) y del sufrimiento quijotesco (v.15). Y si ha logrado ascender hacia ese lugar en donde lo que enaltece a los hombres es el valor para la lucha, Rocinante demuestra que puede cargar sobre sus espaldas ("su espinazo magro y sufrido", v.15) sin desfallecer al símbolo por excelencia de España, don Quijote. Un 
don Quijote que, como ha analizado Pedro Laín Entralgo, representa el nuevo papel de España y la reactualización de su misión histórica, de una España deseada (1975: 255) y que el hablante lírico convoca bajo el signo de la esperanza universal (v.18). Todo ello comprobaría que, a pesar de que no tiene un origen nobiliario, privación que ha superado con creces, Rocinante se ha ganado su nombre, ha triunfado sobre el origen incierto que pesaba sobre él. El verso final (v.19), que retoma la carencialidad apuntada, marca precisamente no tanto la contraposición entre la falta de linaje y la nobleza actual de Rocinante, como el asombro del hablante lírico ante un ser anónimo y de origen oscuro que se ha forjado a sí mismo.

A la luz del poema "Saludo", podríamos explicar el autorretrato ofrecido por León Felipe en los poemas "I" y "II" de la sección "Biografías Paralelas". De esta manera, "Saludo" funcionaría como un elogio que sirve para insertar el recorrido biográfico al que éste nos invita en la invocación inicial, bajo la protección y el modelo ofrecido por Rocinante. Se cumple de esta manera la ejemplaridad paradigmática que guiaba a Plutarco en sus Vidas paralelas: dejar constancia de aquellos hombres que se elevaron, mediante sus hazañas e ideales, por encima del resto de los mortales. Este planteamiento remite "a la ejemplaridad de estas vidas como suscitadoras de imitación y de emulación" (Cantarella 1972: 224) y es lo que realiza León Felipe cuando destaca en el poema "I" las similitudes entre la biografía de Rocinante y la suya propia. Con ello, León Felipe se valora no sólo en cuanto hombre que asume los riesgos de su destino, sino también en cuanto poeta que ha conquistado las glorias literarias (Chen 1998: 28-9). León Felipe desea mostrar que su entrada al mundo de las letras y su consiguiente fama se la debe a él mismo, pues al igual que Rocinante, la carencia de raíces y de un pasado son sinónimos del máximo aprovechamiento de su parte. Y el acto de nombrarse recoge esta motivación, cuando León Felipe se inventa un pseudónimo con el cual comienza, ya sea una nueva existencia, ya sea la forja de sí mismo ${ }^{5}$

A la luz de lo anterior, llama poderosamente la atención que en el poema "II", León Felipe nos proponga un autorretrato cuyo punto de comparación es directamente "Saludo" y que responde al modelo "Je ne vous raconterai pas ce j'ai fait, mais je vais vous dire qui je suis" (Beaujour 1980: 443). Es como si el autorretratista León Felipe se viera en la obligación de justificar no tanto la producción del discurso sobre sí mismo, sino más bien la posibilidad de materializar la percepción que posee en la comparatio inicial, lo cual confirmaría las transformaciones del sujeto y su triunfo sobre el azar, el olvido y el silencio (Beaujour 1980: 444 y 451). La similitud de una historia común, tal y como se desarrolla en el poema "I", permite allanar el camino hacia un encuentro con Rocinante, de manera que las relaciones, en el circuito de comunicación, desembocan en una singularización que acerca a Rocinante, "el caba110 más 'pura sangre' de la gran mitología española" (v.9, poema "I") a un León Felipe, humano y mortal. Así, el inicio del poema "II" arranca ahí en donde termina precisamente "Saludo":

No tengo pedigree como tú, pero no tengo tampoco ¡ay!

la Historia, la Pre-historia,

la deslumbrante Pre-historia que tú tienes:

La carencia de una estirpe ("No tengo pedigree") sirve de conector catafórico 6 , pues sitúa textualmente la enunciación del hablante lírico y favorece la coherencia que busca la comparatio, 
haciendo que la ausencia de un linaje y la privación del reconocimiento social que aquélla implica sean el germen del autorretrato. Por eso, aunque "pedigree" se emplea como prueba para establecer la genealogía de los animales de raza, aquí funciona como una marca de humanidad que señala un destino común. Sin embargo, el reconocimiento de esta situación carencial desemboca en un lamento (v.2) que dramatiza aquello que separa al hablante lírico de su destinatario. En este sentido, la conjunción adversativa "pero" refuerza el vacío del sujeto y traduce la contrariedad que opone la biografía de Rocinante a la suya, en donde "pedigree" forma un paralelismo con "la deslumbrante Pre-historia" (v.4), cuya explicación se agrega en seguida:

Tú eres el caballo más famoso (v.5)

de nuestra constelación solar.

Sales en la cuadriga de Apolo todas las mañanas

por los altivos cerros de Oriente...

De esta manera, el hablante lírico subraya el destino lumínico, por lo cual el "pedigree" de Rocinante resalta su linaje de alcurnia y la fama que se ha ganado por sus hazañas y sus grandes empresas. Esto explicaría el sentido que adquiere la "Historia" con mayúscula (v.3); su selección justificaría el elogio solemne y las funciones excelsas que revelan la clase de caballo que es Rocinante. Al identificar a Rocinante con poderes superiores y numinosos, como en los versos 5 al 8, en donde se retoma el motivo del caballo revestido de majestad solar ya analizado en "Saludo", el hablante lírico interpreta la historia de Rocinante en términos cuasi-divinos. Aunque esto hace posible la contemplación lírica, dotar a Rocinante con estos atributos y a su existencia con tal reverencia, el elogio obliga al hablante lírico a un reconocimiento ineludible:

En esto nos diferenciamos, Rocinante...

Bien lo sé. (v.10)

Tú irás al cielo o al Olimpo, donde quieras.

Yo no tengo asegurada aún mi gloria.

Al señalar la no identidad como núcleo de la comunicación lírica, el poema se dirige hacia el principio de alteridad como constituyente del ser del poeta: León Felipe es en relación con Rocinante y en oposición a lo que es él; el valor diferencial emerge para determinar los posibles nexos entre el hablante lírico y el personaje del Quijote. Si la vocación a la Poesía es interpretada en términos de un camino de aprendizaje y de una revelación mística en Ganarás la luz (Chen 1999), en Rocinante adquiere otro rasgo complementario como vía de promoción social? He ahí en donde las vidas se separan y se desarrollan por vías a veces sinuosas que determinan la existencia. Rocinante, gracias a la fortuna y a la fama alcanzada por el Quijote, tiene un futuro asegurado en los cielos del Olimpo, signo de su ascensión espiritual y de la condición que ostenta (v.11); mientras que el hablante lírico debe aún alcanzar la fama y la honra. Encontramos aquí reproducido el dinamismo contradictorio que, según Françoise Peyrègne, opone las aspiraciones del poeta y el destino de los hombres, "bajo la forma de un doble impulso opuesto y simultáneo hacia arriba y hacia abajo" (1983: 201): hacia arriba está Rocinante; hacia abajo se encuentra él todavía forjándose ${ }^{8}$. Además, la carencia de "gloria" ubica al hablante lírico en la perspectiva de un proceso aún inconcluso, en la apremiante necesidad de seguir comprendiéndose a sí mismo y "con la tentativa de quien está explorando el significado de su biografía personal" (Navajas 
1993: 125-6) ${ }^{9}$. Lo anterior permite al sujeto distanciarse de sí mismo en una estrategia que intenta neutralizar las críticas en su contra y que él reproduce en el inconformismo del poeta (Sánchez 1984b: 194):

\author{
Y las grandes mafias aristocráticas de México \\ dicen cuando me ven pasar: \\ Ahí va esa daga desdentada (v.15) \\ que no ha logrado encontrar todavía \\ ni una perla \\ ni una pepita de oro \\ en las turbias y profundas aguas del surrealismo... \\ ¡Pobre León-felipe... sin perla y sin pepita! (v.20)
}

En el momento en que León Felipe asume lo que otros dicen acerca de él, surge precisamente aquello que acerca Rocinante al hablante lírico y el calificativo de "daga desdentada" precisa tales coincidencias. Recordemos que "daga" es una espada antigua, cuya hoja, en este caso, no posee el filo adecuado para cortar; pero la "daga desdentada" (v.15) no solo se refiere a Rocinante, jamelgo sin dientes como apunta el Quijote, sino también al hablante lírico, un hombre viejo al cual le falta también su dentadura. La situación inicial de carencia emerge de nuevo para enfatizar, además del origen, las similitudes físicas y es aquí en donde el poema se cierra circularmente sobre la reivindicación de la identidad, haciendo de la negación su más atrevido argumento de defensa. La ironía del poema descansa en la asunción de los calificativos de las "mafias aristocráticas" (v.13) en el último verso, con lo cual el hablante lírico los desarticula burlándose de ellos. Si como éstas dicen, el hablante lírico es un viejo que anda buscando todavía los dientes que se le han caído ("sin perla") y, principalmente, las glorias literarias en el mar turbulento de la poesía ("sin pepita"), León Felipe aborda esto sin ningún complejo y lo asume como diferencia sustancial. Su estrategia no se hace esperar y para aquellos que le desean el mal y no reconocen su trabajo, pone el ejemplo del oscuro y viejo Rocinante, quien se forja un destino lumínico y se labra, gracias a Cervantes, una fama universal. Comprendemos, entonces, el sentido que adquiere la invocación de Rocinante en "Saludo"; el hablante lo interpela para que no sólo venga en su auxilio contra críticas mal fundadas, sino que también dinamiza su cercanía solidaria con el caballo más famoso de la "españolidad"10.

\title{
Notas
}

1. León Felipe insiste en esta interpretación de su destino, cuando nos presenta su vocación poética como un camino de aprendizaje y de perfección al que es llamado por una fuerza sobrenatural, la Poesía, que lo obliga a comprometerse en el dolor y el sufrimiento con todos los españoles. Por eso, asume en Ganarás la luz la funciones de un profeta y se identifica con el profeta Jonás (Chen 1999).

2. Observemos lo que dice el texto cervantino: "Fue luego a ver su rocín, y aunque tenía más cuartos que un real y más tachas que el caballo de Gonela, que tantum pellis et ossa fuit, le pareció que ni el Bucéfalo de Alejandro ni Babieca el del Cid con él se igualaban [...] y así, después de muchos nombres que formó, borró, añadió, deshizo y tornó a hacer en su memoria e imaginación, al fin le vino a llamar Rocinante, nombre, a su parecer, alto, sonoro y significativo de lo que había sido cuando fue rocín [...]" (Cervantes 1979: 21, las cursivas son del autor. Tomo I, Cap. I). 
3. Originalmente el caballo se relaciona con las tinieblas y con lo instintivo. Es ante todo un símbolo del mundo ctónico, portador de la muerte y de la vida (Chevalier 1993: 208).

4. Al respecto agregan Chevalier y Gheerbrant: "Vestido con blaca vestimenta de majestad, deja [el caba1lo] entonces de ser lunar y ctónico y se convierte en uránico o solar, en el país de los dioses buenos y los héroes [...] Este blanco caballo celeste representa el instinto controlado, dominado, sublimado; según la ética nueva, es la más noble conquista del hombre" (1993: 208-9).

5. En los versos 22 al 32 del poema "I", León Felipe enuncia esta toma de conciencia:

"A mí no me confirmó una orden tan famosa como la tuya,

pero yo lo que quería

era llamarme León-felipe,

que me llamasen todos León-felipe.

Me parecía entonces un nombre mágico

que alguna vez se había escrito

con lágrimas y sangre.

No era, desde luego,

un nombre tan bonito como el tuyo.

Hoy quiere decir:

antes y después de todos los poetrastos del mundo" (1982: 18-9).

Para un análisis detallado de este poema, remito a mi artículo Chen 1998.

6. Es decir, sirve de conexión con el texto que precede, con algo ya mencionado y que se encuentra topicalizado en el contexto, gracias a una propiedad del individuo que debe ser conocida.

7. En el caso de Rocinante, estos dos serían los principios estructuradores del texto, en donde el yo se construye como disponibilidad y como virtualidad para actuar sobre sí mismo.

8. Françoise Peyrègne señala que a este dinamismo contradictorio corresponde una concepción de las relaciones entre la vida y la historia en la que ambas categorías "están sometidas a violentos impulsos ascendentes y descedentes" (1983: 202).

9. Con ello, por otra parte, el hablante lírico pretende situarse en un punto más genuino frente a la contingencia de la existencia y de su proceso de autoconocimiento.

10. No cabe duda de ello, si nos detenemos en la atracción que podría suscitar el origen etimológico del nombre "Felipe", que viene del griego y significa "el amante de los caballos". La motivación onomástica ayuda a comprender la selección de Rocinante y no la de don Quijote a la hora de establecer su comparatio. Por otro lado, en los poemas de la sección IMD "La intrépida metáfora demiúrgica", el elogio se extiende a Cervantes y a don Quijote en el marco de la españolidad abiertamente exaltada.

\section{Bibliografía}

Bajtín, Mijail. 1982. Estética de la creación verbal. México, D.F.: Siglo Veintiuno Editores.

Beaujour, Michel. 1980. "Autographie et autoportrait”. Poétique. 44, 442-58.

Cantarella, Raffaele. 1972. La literatura griega a la época helenística e imperial. Buenos Aires: Editorial Losada. 
Chen Sham, Jorge. 1997. "Vidas paralelas en la historia de la recepción cervantina: Gregorio de Mayans y Miguel de Unamuno". Káñina, Revista de Artes y Letras de la Universidad de Costa Rica. 21 (2, número especial): 9-15.

1988. "León Felipe a la luz de su historia: la reivindicación recapituladora y autobiográfica en el poema "I" de Rocinante". Revista de Filología y Lingüística de la Univesidad de Costa Rica. 24(1): 23-32.

1999. "El hombre redivivo: el poeta Jonás y su camino de aprendizaje en León Felipe". Letras de Deusto. 29 (84), por aparecer.

Chevalier, Jean y Alain Gheerbrant. 1993. Diccionario de los símbolos. 3a. edición. Barcelona: Editorial Herder.

Genette, Gérard. 1987. Seuils. París: Editions du Seuil.

Kayser, Wolfgang. 1981. Interpretación y análisis de la obra literaria. 5a. reimpresión. Madrid: Editorial Gredos.

Laín Entralgo, Pedro. 1975. La generación del noventa y ocho. 8a. edición. Madrid: Editorial Espasa-Calpe.

León Felipe. 1982. Ganarás la luz. Madrid: Editorial Cátedra (edición a cargo de José Paulino).

1982. Rocinante. Madrid: Editorial Visor.

Madariaga, Salvador de. 1972. Guía del lector del "Quijote": ensayo psicológico sobre el "Quijote". Buenos Aires: Editorial Sudamericana.

Navajas, Gonzalo. 1993. "Una estética para después del postmodernismo: la nostalgia asertiva y la reciente novela española". Revista de Occidente. 143: 105-30.

Ortega y Gasset, José. 1990. Meditaciones del Quijote. 2a. edición (a cargo de Julián Marías). Madrid: Editorial Cátedra.

Peyrègne, Françoise. 1983. "Las metáforas del dinamismo en León-Felipe". Cuadernos Americanos. 249 (4): 199-215.

Rivera, Tomás. 1973. "La teoría poética de León Felipe". Cuadernos Americanos. 186 (1): 193-214.

Sánchez, Alberto. 1984a. "Los ensayos cervantinos de León Felipe". Ínsula. 452-3, 4. 
Sánchez, Alberto. 1984a. "Los ensayos cervantinos de León Felipe". Ínsula. 452-3, 4. 1984b. "Cervantismo y quijotismo de León Felipe". Anales Cervantinos. 22: 181-98.

Starobinski, Jean. 1974. La relación crítica (Psicoanálisis y Literatura). Madrid: Taurus Ediciones.

Unamuno, Miguel de. 1966. Obras completas. Tomo I. Madrid: Editorial Escelicer. 1990. Vida de Don Quijote y Sancho. México D.F.: REI. 